\title{
Ultrasound in Contemporary Physiotherapy Practice
}

\section{Ultrasonics}

Special Issue

\author{
Tim Watson \\ Professor of Physiotherapy \\ University of Hertfordshire
}

t.watson@herts.ac.uk

www.electrotherapy.org

\section{Scope}

Although therapeutic ultrasound has been used for over 50 years in physiotherapy, its use in the clinical environment has changed significantly over this period, and whereas in the past, its use was primarily for its thermal effect, it is now more widely employed for its 'non thermal' effects, especially in relation to tissue repair and wound healing.

There is a substantial volume of published evidence relating to the effects and use of ultrasound as a therapeutic modality, and the overall aim of this paper is to review the current use of ultrasound in the realm of physiotherapy and associated practice, providing an overview of key issues. Whilst considering the evidence and providing key reference material, it does not purport to be a systematic review of the literature. 


\section{Historical use and recent developments}

Ultrasound is almost certainly the most widely used of the electrophysical agents in current clinical practice. In addition to its widespread use by physiotherapists [1-3], it is also commonly used by numerous therapists from other professional groups (e.g. osteopaths, chiropractors, sports therapists). The results of a recent national survey of physiotherapists carried out in Australia [4] indicates that therapeutic ultrasound remains the most popular modality in use.

The results of a survey carried out in Britain in 1985 [2] showed that $20 \%$ of all physiotherapy treatments in NHS departments and $54 \%$ of all private treatments involved therapeutic ultrasound and the widely cited survey by Pope et al [1] identified ultrasound as the most frequently employed modality (94\%) and $64 \%$ of respondents reported that they used the modality more than once a day.

In the 1985 survey, it was shown that there were large variations in the use of ultrasound including a range of intensities from $0.1-3.0 \mathrm{~W} / \mathrm{cm}^{2}$ giving a variation factor of 30 from the lowest to the highest applied intensity. The current application of ultrasound for fracture healing at even lower doses (typically $0.03 \mathrm{~W} / \mathrm{cm}^{2}$ ) would take that to a factor of 100 . This is a very substantial variation on just one factor that affects the output of the machine, and it is not surprising therefore that some research evidence is supportive of the modality whilst other publications are clearly not. Given that the effects of 'electrotherapy' interventions have a dose dependency [5], this wide variation in applied power would be expected to generate a range of effects including more and less effective therapeutic outcomes. Further identification of the critical machine and dose parameters is clearly needed and is being undertaken within this research group.

\section{Physics Related Issues}

The physics of therapeutic ultrasound are outwith the remit of this paper, but there are two essential issues that have a direct influence of practice and will therefore be identified in that context. 


\section{Coupling Media}

Ultrasound will be reflected at the metal/air interface found at the treatment head, it is necessary to provide a medium through which the ultrasound can freely pass in order to reach the patients tissues. This medium is most commonly referred to as a coupling medium, and several different types are used in practice. Given that the job of the coupling medium is to allow transmission of the ultrasound, a coupling medium that absorbs, changes or disturbs the ultrasound energy is not performing in an ideal way.

The coupling media used in this context include water, various oils, creams and gels. Ideally, the coupling medium should be sufficiently fluid to fill all available spaces, relatively viscous so that it stays in place, have an impedance appropriate to the media it connects, and should allow transmission of ultrasound energy with minimal absorption, attenuation or disturbance. For extensive discussions regarding coupling media, see [6-10]. Water and gel based media are clearly preferable to oil and cream based media. A recent detailed study considering the effect of different coupling gels on ultrasound transmission did demonstrate that there were differences in transmission characteristics and absorption levels of commonly employed ultrasound gels [9] but that there was no clinically significant difference between them. Most of the commonly employed gels only varied in their absorption characteristics by approximately $3 \%$ compared with water, and given the inaccuracy of clinical machine calibration [11], this is an insignificant variability. The addition of drug based material to the gel (for the purpose of phonophoresis) is not strongly supported by the literature, and the preliminary results from ongoing work in this unit (Todd and Watson) would suggest that the inclusion of pharmaceutical agents in the ultrasound gel appears to significantly reduce ultrasound transmission to the tissues, and therefore reduces the efficacy of the ultrasound component of the treatment. Whether this is outweighed by the clinical benefits of enhanced drug delivery remains to be established.

In an extension to this work, the capacity for various wound dressing materials to transmit ultrasound has been investigated. If it can be argued that of ultrasound energy can be effectively passed through a wound dressing without significant absorption, there is a potential value for not having to disturb the dressing in order to apply this modality which has an established role in chronic wound management [1215]. Furthermore, US scanning of the wound bed and environment without having to remove the dressing may be clinically advantageous, especially given that the removal of wound dressings is considered to result in inhibited repair for several hours on each occasion [16]. 
A total of 48 different wound dressings were evaluated, and there was a very wide variation in their transmission characteristics, ranging from excellent to zero [17]. The dressing included alginates, foams, honey-impregnated dressings, hydrocolloids, hydrogel sheets, low-adherence dressings, vapour-permeable films and odourabsorbent dressings. In practice multiple dressings may be applied to a wound, for example an alginate held in place with a vapour permeable film. If ultrasound were to be applied through these dressings, a layer of standard couplant gel might be applied. Therefore additional tests were carried out on combination of 2 typical dressings plus couplant gel to see if the overall transmissivity was significantly different from what would be expected by combining the data gathered separately for each component. Some dressings were found to behave in an unstable fashion (demonstrating visible changes in the structure of the dressing material) when using a continuous mode of ultrasound and these were further tested with the energy applied in a pulsed mode.

The results demonstrate that many of the dressings tested are good transmitters of ultrasound which suggests that there may be scope, previously unexplored, for the application of therapeutic US to wounds through a variety of dressings. However the data also demonstrate that it is not appropriate to make generalisations about dressing transmissivity. Each dressing has its own transmission characteristics, which may change with both US power and frequency. Some dressings that were unstable using a continuous beam demonstrated stability when the duty cycle was reduced to $20 \%$, suggesting that a thermal mechanism may be involved as US energy absorbed by the dressing increased its temperature and may have caused its acoustic characteristics to change.

The dressings tested vary significantly in their capacity to transmit ultrasound, and some demonstrate excellent US transmissivity characteristics. Even dressings of the same type had significantly different transmissivities. This may be of particular importance in the case of hydrogel dressings, since these are recommended as a class of dressings through which wounds may be insonated [18]. The results indicate that their transmissivities may vary between $80 \%$ and $100 \%$. This means that for a given nominal power output by a US generator, there might be considerable disparity in the power reaching the wound according to the specific hydrogel dressing used. Differences within some other classes of dressing are much greater, with transmissivity varying from $100 \%$ to zero. Combination of dressings in layers showed results that were as would have been predicted from the individual sample data. Detailed analysis and ranking of the 48 dressings tested at varying frequency and power output are included in the original paper [17]. 


\section{Absorption}

In order to have a therapeutic effect, absorption of the applied energy is necessary [19], hence the effectiveness of the modality will vary according to a tissues capacity to absorb the applied energy. Tissues with a higher protein content will absorb US to a greater extent, thus tissues with high water content and low protein content (e.g. blood and fat) absorb little of the US energy whilst those with a lower water content and a higher protein content (e.g. ligament, tendon) will absorb US far more efficiently. It has been suggested $[19,20]$ that tissues can therefore be ranked according to their tissue absorption (Figure 1)

\section{INSERT FIGURE}

Figure 1 : Ultrasound tissue absorption characteristics

Although cartilage and bone are at the upper end of this scale, the problems associated with wave reflection at the tissue surface mean that a significant proportion of US energy striking the surface of either of these tissues is likely to be reflected. The best absorbing tissues in terms of clinical practice are those with high collagen content - ligament, tendon, fascia, joint capsule, scar tissue [3, 20-23].

The differential absorption of US energy has an important influence on clinical decision making. Using ultrasound to treat a lesion located in a tissue that is a poor absorber of the energy is less likely to be effective when compared with treating a tissue which is a 'better' absorber of the energy. For example, in a recent paper which evaluated the effectiveness of therapeutic ultrasound immediately following contusion injury in muscle [24] no significant beneficial effects were demonstrated. Although this could be interpreted as a negative research finding, it would be difficult to argue why ultrasound should have an effect following this type of injury as the insulted tissues will absorb a relatively small proportion of the energy and hence it is unlikely to have a significant effect. Trials that have evaluated the effect of the same modality on lesions in dense collagenous tissue have demonstrated significant therapeutic effects (e.g. [25, 26]). 
An important rider to this phenomenon is that once damaged, musculocutaneous tissues will repair which involves the formation of scar tissue, and hence, therapeutic ultrasound may be of significant clinical value in that the scar tissue becomes the target of the therapy. In a damaged muscle, ultrasound could therefore be expected to be effective once the scar tissue has become established.

\section{Thermal vs Non-Thermal}

When ultrasound travels through tissue a percentage of it is absorbed, and this leads to the generation of heat within that tissue. The amount of absorption depends upon the nature of the tissue, its degree of vascularisation, and the frequency of the applied ultrasound. Tissues with a high protein content absorb ultrasound more readily than those with a higher fat content, and also the higher the US frequency, the greater is the absorption rate. A biologically significant thermal effect can be achieved if the temperature of the tissue is raised to between 40 and $45^{\circ} \mathrm{C}$ for at least 5 minutes. Controlled heating can produce desirable effects [27], which include pain relief, decrease in joint stiffness and increased local blood flow. Whilst the therapeutic benefits of tissue heating are well established, ultrasound is relatively inefficient at generating sufficient thermal change in the tissues to achieve this therapeutic effect when applied at commonly applied clinical doses.

Historically, US has been widely employed for its thermal effects, but it has been argued more recently that the 'non thermal' effects of this energy form are more effective $[3,19]$ and currently, the majority of clinical applications focus on these. Gallo et al [28] demonstrated that both continuous and pulsed ultrasound interventions generated measurable thermal changes in the tissues (muscle), but considering the temperature changes achieved, they would be expected to be of minimal therapeutic value. Garrett et al [29] compared the heating effect of a pulsed shortwave treatment with an ultrasound treatment, and the pulsed shortwave intervention was clearly more effective at achieving the required thermal change for therapeutic benefit. A patient treated with ultrasound in continuous mode at relatively high power density will feel a temperature change (mainly in the skin which is where thermal receptors are predominantly located), but this is not the same as achieving a clinically significant thermal change in deeper tissues.

A recent comparative study from this research unit evaluated the efficacy of a locally applied thermal modality with (kilohertz) ultrasound with regards their effect on soft 
tissue extensibility [30]. Although both modalities were found to be effective in this regard, the ultrasound demonstrated no significant benefit over the heat treatment, and in fact, there was a trend for the thermal intervention producing greater benefit. In another ultrasound, heating and stretching study, Draper and Ricard [31] demonstrated that there was a clinically useful temperature rise in the tissues but that the return to baseline was swift, and the therapeutic window for stretching following US application was limited to some 3 minutes immediately after treatment. Although there are certainly ultrasound generated thermal changes in the tissues, it is important to differentiate between those produced in the skin and superficial tissues - where they are easily detected by the patient - and those generated at 'depth' which are of more clinical importance, and are not readily identifiable by the recipient of the therapy. An example of the skin surface thermal changes are seen in Figure 2. Ultrasound was applied for 5 minutes at $1.5 \mathrm{~W} \mathrm{~cm} 2$ in continuous mode using a $3 \mathrm{MHz}$ application over an area of twice the treatment head. The change in peak temperature in the central hand zone was from $34.3 \mathrm{C}$ to $37.3 \mathrm{C}$ by the end of the application. Clearly, this is more than sufficient for the patient to report a feeling of warmth or heating, but that does not necessarily equate to a therapeutic heating at any tissue depth.

\begin{tabular}{|l|l|}
\hline & \\
\hline (a) Baseline thermograph & $\begin{array}{l}\text { (b) Thermograph at the end of the 5 } \\
\text { minute treatment period }\end{array}$ \\
\hline
\end{tabular}

Figure 2 : Thermographic images of the hand before and after ultrasound

Ultrasound has historically been used primarily for its perceived thermal effects, and although this is a less frequently employed rationale in current clinical practice, there remain many therapists for whom the heating effects are considered to be the most important. Tissue heating is clearly of value in numerous clinical conditions, but the evidence does not fully support the use of ultrasound as an efficient thermal intervention. There are tissue heating methods available in clinical practice that are better at achieving the desired thermal changes and based on the available evidence, should be used in preference to ultrasound.

There are many situations where ultrasound produces bioeffects and yet significant temperature change is not involved (e.g. low spatial-average temporal-average (SATA) intensity). It is not strictly true to talk about 'non-thermal' mechanisms, in that the delivery and absorption of energy in the tissues will result in a temperature rise. 
The term 'non-thermal' in this context relates to the fact that there is no apparent thermal accumulation in the tissues, and is sometimes now referred to as a 'microthermal' effect [19, 32]. The physical mechanisms thought to be involved in producing these non-thermal effects include cavitation and acoustic streaming [10, 22, 33].

There is evidence indicating that 'non-thermal' mechanisms play a primary role in producing a therapeutically significant effect and the focus of this section will relate to these effects in relation to soft tissue injury as it is the most commonly employed in physiotherapy clinical practice.

\section{Ultrasound in Soft Tissue Repair}

The process of tissue repair is a complex series of cascaded, chemically mediated events that lead to the production of scar tissue that constitutes an effective material to restore the continuity of the damaged tissue. The process is more complex than be described in this paper, but have been extensively reviewed elsewhere, including [34].

The effect of US during the repair process varies according to the primary events that are occurring in the tissues. Immediately following injury, during the tissue bleeding phase, it is generally considered to be inadvisable to use ultrasound in that if it has the capacity to enhance local blood flow, there would be a clinical disadvantage to doing so whilst tissue bleeding was occurring. Once active bleeding has stopped, it is appropriate to start using ultrasound as soon as is feasible. During the inflammatory phase, US has a stimulating effect on the mast cells, platelets, white cells with phagocytic roles and the macrophages [22, 35-37]. For example, the application of ultrasound induces the degranulation of mast cells, causing the release of arachidonic acid which itself is a precursor for the synthesis of prostaglandins and leukotreine - which act as inflammatory mediators [25, 37, 38]. By increasing the activity of these cells, the overall influence of therapeutic US is certainly pro-inflammatory rather than anti-inflammatory. The benefit of this mode of action is not to 'increase' the inflammatory response as such (though if applied with too greater intensity at this stage, it is a possible outcome [39], but rather to act as an 'inflammatory opimiser'. The inflammatory response is essential to the effective repair of tissue and inhibition of these events serves to inhibit the repair phases that follow [34, 40-42], and the more efficiently the process can complete, the more 
effectively the tissue can progress to the next phase (proliferation). Studies which have tried to demonstrate the anti inflammatory effect of ultrasound have largely failed to do so (e.g.[43, 44], and have suggested that US is ineffective. It is effective at promoting the normality of the inflammatory events, and as such has a therapeutic value in promoting the overall repair events [19, 22].

Employed at an appropriate treatment dose, with optimal treatment parameters (intensity, pulsing and time), the benefit of US is to make the earliest repair phase as efficient as possible, and thus have a promotional effect on the whole healing cascade. For tissues in which there is an inflammatory reaction, but in which there is no 'repair' to be achieved, the benefit of ultrasound is to promote the normal resolution of the inflammatory events, and hence resolve the 'problem' This will of course be most effectively achieved in the tissues that preferentially absorb the energy.

During the proliferative phase (scar production) US also has a stimulative effect (cellular up regulation), though the primary active targets are now the fibroblasts, endothelial cells and myofibroblasts [21, 36-38, 45-48]. These are all cells that are normally active during scar production and US is therefore pro-proliferative in the same way that it is pro-inflammatory - it does not change the normal proliferative phase, but maximises its efficiency - producing the required scar tissue in an optimal fashion. Harvey et al [49] demonstrated that low dose pulsed ultrasound increases protein synthesis and several research groups have demonstrated enhanced fibroplasia and collagen synthesis [46, 50-54]. There is currently a growing interest in the potential role for ultrasound therapy in relation to the angiogenic response which is an essential component of the proliferative phase, and future research may serve to identify additional therapeutic benefits which at the moment remain largely speculative, or based solely on laboratory based research (e.g. [55]). Transfer to the clinical environment has yet to be established.

Scar tissue is an essential component of the repair, and for most musculoskeletal tissues, it is the best outcome that can be achieved. In many ways, tissue regeneration would be ideal, but is not available in most musculoskeletal tissues. Functional scar tissue may be considered to be second best - but the aim of therapy should be to promote the construction of the most efficient scar possible.

During the remodelling phase of repair, the somewhat generic scar that is produced in the initial stages is refined such that it adopts functional characteristics of the tissue that it is repairing [34]. A scar in ligament will not 'become' ligament, but will 
behave more like a ligamentous tissue. This is achieved by a number of processes, but mainly related to the orientation of the collagen fibres in the developing scar and also to the change in collagen type, from predominantly Type III collagen to a more dominant Type I collagen [56]. The remodelling process is certainly not a short duration phase - research has shown that it can last for a year or more - yet it is an essential component of quality repair and the application of ultrasound appears to enhance the events normally associated with this phase.

Ultrasound can influence the remodelling of the scar tissue in that it appears to be capable of enhancing the appropriate orientation of the newly formed collagen fibres and also to the collagen profile change from mainly Type III to a more dominant Type I construction, thus increasing tensile strength and enhancing scar mobility (e.g. [21]). Ultrasound applied to tissues enhances the functional capacity of the scar tissues [21,53]. The role of ultrasound in this phase may also have the capacity to influence collagen fibre orientation as demonstrated in an elegant study by Byl et al [57], though their conclusions were quite reasonably some tentative. Other studies have demonstrated effects of ultrasound therapy on collagen behaviour in this latter repair stage (e.g. [26, 58-60]).

The application of ultrasound during the inflammatory, proliferative and repair phases is not of value because it changes the normal sequence of events, but because it has the capacity to stimulate or enhance these normal events and thus increase the efficiency of the repair phases [19,22]. It would appear that if a tissue is repairing in a compromised or inhibited fashion, the application of therapeutic ultrasound at an appropriate dose will enhance this activity. If the tissue is healing 'normally', the application will, it would appear, speed the process and thus enable the tissue to reach its endpoint faster than would otherwise be the case. The effective application of ultrasound to achieve these aims is dose dependent $[5,59]$ though some authors have challenged the reality of dose dependency in this regard (e.g. [61]).

\section{Ultrasound in Fracture Repair}

Low intensity pulsed ultrasound (LIPUS) has emerged as an effective therapeutic approach in fracture repair, for both fresh fractures and those experiencing inhibited repair. Whereas previously, a fracture has been considered to be a local contraindication to therapeutic US, the current evidence demonstrates that this is clearly not the case. The review of this material is covered in a separate paper and will not therefore be considered in detail here. It is likely that the further development of this therapy will influence clinical practice, though in many instances it is not 
necessary for the therapist to 'do' the treatment, and patient self management appears to be the most effective way forward in this area. Whether a standard therapy ultrasound machine is capable of effectively delivering the required dose is still not resolved. Issues relating to accurate dose (intensity) delivery and the relatively high Beam Nonuniformity Ratios (BNR) of current clinical devices raise some concern, though it has been proposed that it might be a realistic proposition (e.g. $[62,63])$

\section{Clinical Issues}

\section{Machine Calibration}

Concern has been raised with regards the accuracy of clinical ultrasound machines. When used in research programmes, operating machine calibration is (normally) checked to ensure accuracy, this is less likely to be the case in practice. Even if a machine undergoes an annual check, this may not include a calibration check and Pye and Milford [11] in an evaluation of machines used in NHS practice in Scotland identified almost $70 \%$ of machine outputs that differed from the expected value by more than $30 \%$. More accurate test and calibration procedures have been proposed, but at the current time, it is likely that a significant proportion of machines in use are not delivering what the therapists assumes (based on the output set or shown on the device). Weekly checks using simple balance meters may help to identify gross inaccuracies, but a more comprehensive and rigorous quality assurance system is almost certainly required. Further papers [11,64] have identified and tried to quantify the risks associated with this problem and more recently [65] have reported a cost effective method for measuring machine output accurately.

\section{Potential Infection}

Infection risk is a topical issue in current practice, and the possibility of passing on infective micro-organisms as a result of healthcare events is a growing area of concern. Clearly, there is a clinical risk associated with any intervention, but these need to be minimised as far as possible. When using ultrasound as part of a wound management programme, practitioners are usually mindful of infection risk and take careful and adequate precautions. In 'normal' clinical practice, it is less common to find cleaning of the ultrasound treatment applicator between every treatment, though many departments do have comprehensive infection control policies and routinely clean the machines and applicators. 
A recent study [66] investigated whether clinical ultrasound units were a potential vector of infection. The study aimed to determine the degree of contamination on therapeutic ultrasound transducer heads and ultrasound gel after routine clinical use, and to evaluate the efficacy of recommended infection control procedures. The study was conducted in Australia and microbiological cultures were obtained from 44 transducer heads and 43 gels (machines were in use in hospitals, private practices and other healthcare facilities). Swabs taken from the treatment heads and the gel bottles were cultured and colony growth assessed. Twenty-seven per cent of transducer heads and $28 \%$ of gels were contaminated. Transducer heads showed fairly low levels of contamination across the sample, with the majority of organisms isolated found in normal skin and environmental flora. Gels were heavily contaminated with opportunistic and potentially pathogenic organisms, including Stenotrophomonas maltophilia, Staphylococcus aureus, Acinetobacter baumannii and Rhodotorula mucilaginosa. No multi-resistant organisms were identified.

A second element of the study was to evaluate the effectiveness of cleaning the treatment head with $70 \%$ alcohol which significantly reduced the level of contamination on transducer heads. The authors conclude that routine cleaning of the treatment head with $70 \%$ alcohol between treatments should be the clinical 'norm', and although this does not deal with the micro-organism colonies in the gel bottles sampled, it is at least a significant move in the right direction, and is currently being advocated as best practice.

Re-usable gel containers are likely to be a bigger problem in this regard, and if large volume gel containers are purchased (for gains in cost saving), it is unlikely to be good practice to keep refilling the same small bottles for use in the department. At the very least, either new or sterilised clean bottles should be used to reduce the risk until further research and management strategies have been identified.

\section{Dose Issues}

The widely used term 'treatment dose' of an ultrasound application is used in the clinical environment as a combination of the selectable machine parameters frequency, power density, duty cycle and treatment time, thus has a more casual meaning than in the classic physics sense. 
Whilst it is beyond the scope of this review to consider dosing issues in detail, there are several general points that merit brief consideration. Firstly, there does appear to be a dose dependency in relation to therapeutic effects [5], though as identified previously, this has been challenged in the literature [61]. There are a myriad of possible combinations of frequency, power, power density, duty cycle and treatment times and from the published evidence it appears that there are some combinations that are more effective than others. There is not an exhaustive range of clinical literature from which to make absolutely definitive suggestions, but the trends that have emerged are sufficiently strong to justify a dose decision making model that is effective and amenable to adjustment as new research comes to light [67].

Given the growing evidence for therapeutic windows in most, if not all areas of practice, the main concern is to try and identify which of the dose parameters that can be manipulated are in fact the most critical and which are less important. Leaving to one side the general decision with regards ultrasound frequency, the power density $\left(\mathrm{W} \mathrm{cm}^{-2}\right)$, duty cycle and total treatment time appear to be fundamental. Although there is a trend for outcomes to be related to the actually duty cycle $(20 \%, 25 \%, 33 \%$ etc), the more basic decision is whether to apply the energy in a continuous or pulsed mode. The continuous mode will result in more rapid delivery of the desired energy, but there is a strong, and growing body of evidence that support the contention that pulsed mode ultrasound is more effective than continuous mode, especially in relation to tissue repair, and most critically, in the early (inflammatory and proliferative) stages.

An additional issue relates to current clinical practice and treatment frequency. Much of the research that has demonstrated significant clinical benefit has employed treatments at regular and relatively frequent intervals. Due to real world restrictions in current healthcare practice, many patients are seen regularly but infrequently (for example once every 2 or 4 weeks). If ultrasound is used when the patient attends on this basis, there is no evidence that there is a clinically significant gain - there may be, but this is a case of a lack of evidence as opposed to evidence of a lack of effect - an area needing some urgent consideration.

Further evaluation of effective doses is being undertaken in this research unit, and it is anticipated that a more comprehensive dose model will emerge as a result. 


\section{Overlap between Modalities}

At face value, the therapeutic effects of ultrasound are remarkably similar to those of laser therapy and some pulsed EM fields. Given that all three are means of delivering energy such that cellular up-regulation results, this is actually not surprising. The fundamental difference relates back not to a difference in their basic effect, but more strongly to the fact that these three energies are preferentially absorbed in different tissues [19]. There do appear to be some variations between the physiological effects of these interventions, but for the most part, they appear to overlap. Ultrasound is most effective at achieving these effects in the tissues that absorb then mechanical energy, and as described above, these are essentially the dense collagenous tissues. The modality is less effective when it comes to dealing with therapeutic gains in tissues like muscle, nerve and where there is significant oedema. The other modalities appear to offer advantages in these other tissues, and hence, the use of laser, pulsed EM fields (mainly shortwave) and ultrasound are regarded as being complementary rather than competitive in clinical practice.

\section{Summary}

Therapeutic ultrasound has a long history of use in physiotherapy practice, though the emphasis has changed over the last 15-20 years. Several other professional groups are also using the modality including osteopaths, chiropractors, podiatrists and sports therapists. The current emphasis of use relates to enhancement of repair of soft (musculoskeletal) tissues following injury, insult or irritation, and the evidence for its value in these areas is considerable, though there are, of course, gaps that remain and need to be filled with quality research especially in the clinical arena as opposed to the laboratory. 


\section{Figures}

Figure 1 : Ultrasound tissue absorption characteristics

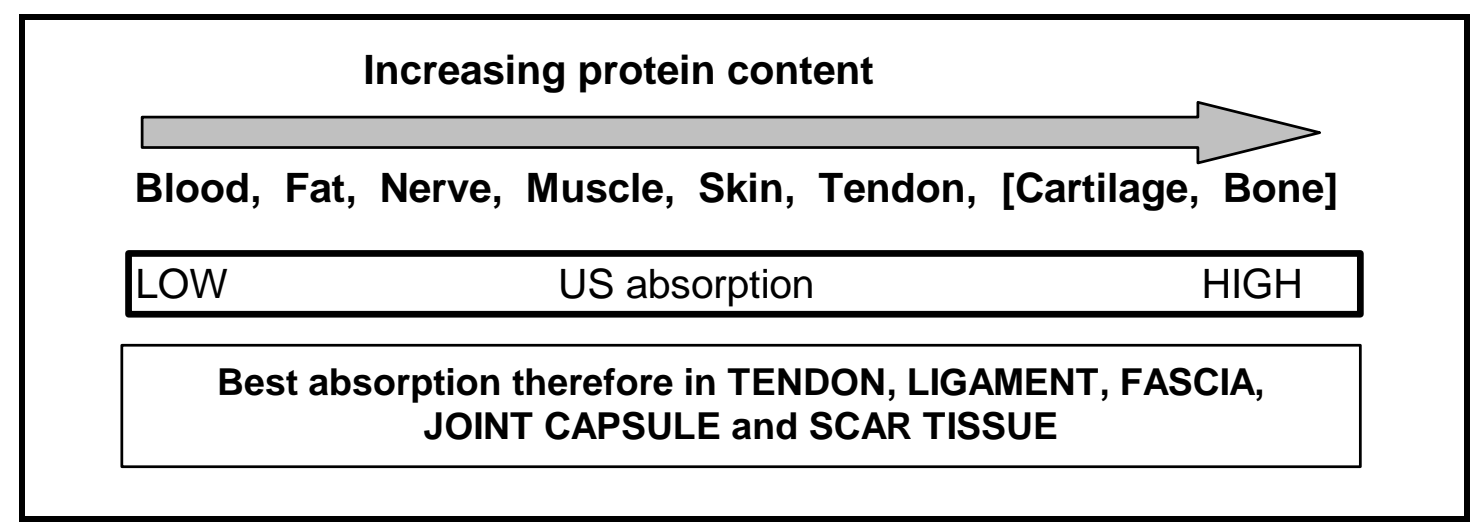

Figure 2 : Thermographic images of the hand before and after ultrasound

(a) Baseline thermograph

(b) Thermograph at the end of the 5 minute treatment period

\section{References}

1. Pope, G.D., S.P. Mockett and J.P. Wright, A survey of electrotherapeutic modalities: ownership and use in the NHS in England. Physiotherapy, 1995. 81(2): p. 82-91.

2. ter Haar, G., M. Dyson and E.M. Oakley, The use of ultrasound by physiotherapists in Britain. Ultrasound in Medicine and Biology, 1985. 13: p. 659663. 
3. Watson, T., The role of electrotherapy in contemporary physiotherapy practice. Man Ther, 2000. 5(3): p. 132-41.

4. Chipchase, L., M. Williams and V.J. Robertson, Electrophysical agents in physiotherapy practice and education: Still a good idea after all these years?, in 15th International World Confederation of Physical Therapy Congress (WCPT). 2007, Physiotherapy 2007;93(S1):S97: Vancouver, Canada.

5. Watson, T. Modality and dose dependency in electrotherapy. in 15th International World Confederation of Physical Therapy Congress (WCPT). 2007. Vancouver, Canada: Physiotherapy 93(S1); S674.

6. Casarotto, R.A., J.C. Adamowski, F. Fallopa and F. Bacanelli, Coupling agents in therapeutic ultrasound: Acoustic and thermal behavior. Arch. Phys. Med. Rehabil., 2004. 85(1): p. 162-165.

7. Docker, M.F., D.J. Foulkes and M.K. Patrick, Ultrasound couplants for physiotherapy. Physiotherapy, 1982. 68(4): p. 124-125.

8. Klucinec, B., M. Scheidler, C. Denegar, E. Domholdt and S. Burgess, Effectiveness of wound care products in the transmission of acoustic energy. Phys Ther, 2000. 80(5): p. 469-76.

9. Poltawski, L. and T. Watson, Relative transmissivity of ultrasound coupling agents commonly used by therapists in the UK. Ultrasound Med Biol, 2007. 33(1): p. 120-8.

10. Williams, R., Production and transmission of ultrasound. Physiotherapy, 1987. 73(3): p. 113-116.

11. Pye, S.D. and C. Milford, The performance of ultrasound physiotherapy machines in Lothian Region, Scotland, 1992. Ultrasound Med Biol, 1994. 20(4): p. 347-59.

12. Flemming, K. and N. Cullum, Therapeutic ultrasound for venous leg ulcers. Cochrane Database Syst Rev, 2000(4): p. CD001180.

13. Flemming, K. and N. Cullum, Therapeutic ultrasound for pressure sores. Cochrane Database Syst Rev, 2000(4): p. CD001275.

14. Hess, C.L., M.A. Howard and C.E. Attinger, A review of mechanical adjuncts in wound healing: hydrotherapy, ultrasound, negative pressure therapy, hyperbaric oxygen, and electrostimulation. Ann Plast Surg, 2003. 51(2): p. 210-8.

15. Johannsen, F., A.N. Gam and T. Karlsmark, Ultrasound therapy in chronic leg ulceration: a meta-analysis. Wound Repair Regen, 1998. 6(2): p. 121-6. 
16. Russell, L., Understanding physiology of wound healing and how dressings help. Br J Nurs, 2000. 8(1): p. 10-12.

17. Poltawski, L. and T. Watson, Transmission of therapeutic ultrasound by wound dressings. Wounds, 2007. 19(1): p. 1-12.

18. McCulloch, J., Physical modalities in wound management: ultrasound, vasopneumatic devices and hydrotherapy. OstomyWound Management, 1995. 41(5): p. 30-2, 34, 36-7.

19. Watson, T., Electrotherapy and tissue repair. Sportex-Medicine., 2006. 29: p. 7-13.

20. Frizzell, L.A. and F. Dunn, Biophysics of ultrasound, in Therapeutic Heat and Cold, J. Lehmann, Editor. 1982, Williams \& Wilkins: Baltimore.

21. Nussbaum, E., The influence of ultrasound on healing tissues. J Hand Ther, 1998. 11(2): p. 140-7.

22. ter Haar, G., Therapeutic Ultrsound. Eur J Ultrasound, 1999. 9: p. 3-9.

23. Watson, T., Current concepts in electrotherapy. Haemophilia, 2002. 8: p. 413418.

24. Wilkin, L.D., M.A. Merrick, T.E. Kirby and S.T. Devor, Influence of therapeutic ultrasound on skeletal muscle regeneration following blunt contusion. Int. J. Sports Med., 2004. 25(1): p. 73-77.

25. Leung, M.C., G.Y. Ng and K.K. Yip, Effect of ultrasound on acute inflammation of transected medial collateral ligaments. Arch Phys Med Rehabil, 2004. 85: p. 963-966.

26. Sparrow, K.J., S.D. Finucane, J.R. Owen and J.S. Wayne, The effects of lowintensity ultrasound on medial collateral ligament healing in the rabbit model. Am J Sports Med, 2005. 33(7): p. 1048-56.

27. Lehmann, J.F. and B.J. DeLateur, Therapeutic heat, in Therapeutic Heat and Cold, J.F. Lehmann, Editor. 1982, Williams and Wilkins: Baltimore, MD.

28. Gallo, J.A., D.O. Draper, L.T. Brody and G.W. Fellingham, A comparison of human muscle temperature increases during $3-\mathrm{MHz}$ continuous and pulsed ultrasound with equivalent temporal average intensities. J. Orthop. Sports Phys. Ther., 2004. 34(7): p. 395-401.

29. Garrett, C.L., D.O. Draper and K.L. Knight, Heat distribution in the lower leg from pulsed short-wave diathermy and ultrasound treatments. Journal of Athletic Training, 2000. 35(1): p. 50-5. 
30. Meakins, A. and T. Watson, Longwave ultrasound and conductive heating increase functional ankle mobility in asymptomatic subjects. Physical Therapy in Sport, 2006. 7.

31. Draper, D.O. and M.D. Ricard, Rate of temperature decay in human muscle following $3 \mathrm{MHz}$ ultrasound: the stretching window revealed. Journal of Athletic Training, 1995. 30(4): p. 304-7.

32. Kitchen, S. and M. Dyson, Low-energy treatments: non-thermal or microthermal?, in Electrotherapy: Evidence based Practice, S. Kitchen, Editor. 2002, Elsevier / Churchill Livingstone: Oxford.

33. Baker, K.G., V.J. Robertson and F.A. Duck, A review of therapeutic ultrasound: biophysical effects. Phys Ther, 2001. 81(7): p. 1351-8.

34. Watson, T., Tissue repair: The current state of the art. Sportex-Medicine., 2006. 28: p. 8-12.

35. Fyfe, M.C. and L.A. Chahl, Mast cell degranulation: A possible mechanism of action of therapeutic ultrasound (Abstract). Ultrasound in Med \& Biol, 1982. 8(Suppl 1): p. 62.

36. Maxwell, L., Therapeutic ultrasound: Its effects on the cellular \& mollecular mechanisms of inflammation and repair. Physiotherapy, 1992. 78(6): p. 421-426.

37. Nussbaum, E.L., Ultrasound: to heat or not to heat - that is the question. Physical Therapy Reviews, 1997. 2: p. 59-72.

38. Mortimer, A.J. and M. Dyson, The effect of therapeutic ultrasound on calcium uptake in fibroblasts. Ultrasound in Med \& Biol, 1988. 14(6): p. 499-506.

39. Ciccone, C., B. Leggin and J. Callamaro, Effects of ultrasound and trolamine salicylate phonophoresis on delayed-onset muscle soreness. Phys Ther, 1991. 71: p. 666-.

40. Bhattacharyya, T., R. Levin, M.S. Vrahas and D.H. Solomon, Nonsteroidal antiinflammatory drugs and nonunion of humeral shaft fractures. Arthritis Rheum, 2005. 53(3): p. 364-7.

41. Hashimoto, I., H. Nakanishi, Y. Shono, M. Toda, H. Tsuda and S. Arase, Angiostatic effects of corticosteroid on wound healing of the rabbit ear. J Med Invest, 2002. 49(1-2): p. 61-6.

42. Tsai, W.-C., F.-T. Tang, C.-C. Hsu, Y.-H. Hsu, J.-W.S. Pang and C.-C. Shiue, Ibuprofen inhibition of tendon cell proliferation and upregulation of the cyclin kinase inhibitor p21CIP1. Journal of Orthopaedic Research, 2004. 22(3): p. 586-591. 
43. El Hag, M., K. Coghlan, P. Christmas, W. Harvey and M. Harris, The antiinflammatory effect of dexamethazone and therapeutic ultrasound in oral surgery. $\mathrm{Br}$ J Oral \& Maxillofacial Surgery, 1985. 23: p. 17-23.

44. Hashish, I., H.K. Hai, W. Harvey, C. Feinmann and M. Harris, Reduction of postoperative pain and swelling by ultrasound treatment: a placebo effect. Pain, 1988. 33(3): p. 303-311.

45. Dyson, M. and D. Smalley, Effects of ultrasound on wound contraction, in Ultrasound Interactions in Biology \& Medicine, R. Millner, E. Rosenfeld and U. Cobet, Editors. 1983, Plenum Press: New York. p. 151-158.

46. Ramirez, A., J.A. Schwane, C. McFarland and B.C. Starcher, The effect of ultrasound on collagen synthesis and fibroblast proliferation in vitro. Med Sci Sports Exerc, 1997. 29: p. 326-332.

47. Young, S. and M. Dyson, Effect of therapeutic ultrasound on the healing of full thickness excised skin lesions. Ultrasonics, 1990. 28: p. 175-180.

48. Young, S.R. and M. Dyson, The effect of therapeutic ultrasound on angiogenesis. Ultrasound Med Biol, 1990. 16(3): p. 261-9.

49. Harvey, W., M. Dyson, J.B. Pond and G. R., The stimulation of protein synthesis in human fibroblasts by therapeutic ultrasound. Rheumatology \& Rehabilitation, 1975. 14: p. 237-.

50. Enwemeka, C.S., The effects of therapeutic ultrasound on tendon healing. A biomechanical study. Am J Phys Med Rehabil, 1989. 68(6): p. 283-7.

51. Enwemeka, C.S., Inflammation, cellularity, and fibrillogenesis in regenerating tendon: implications for tendon rehabilitation. Phys Ther, 1989. 69(10): p. 816-25.

52. Enwemeka, C.S., O. Rodriguez and S. Mendosa, The biomechanical effects of low-intensity ultrasound on healing tendons. Ultrasound Med Biol, 1990. 16(8): p. 801-7.

53. Huys, S., B.S. Gan and M. Sherebrin, Comparison of effects of early and late ultrasound treatment on tendon healing in the chicken limb. J Hand Ther, 1993. 6: p. 58-59.

54. Turner, S., E. Powell and C. Ng, The effect of ultrasound on the healing of repaired cockrel tendon: is collagen cross-linkage a factor? J Hand Surg, 1989. 14B: p. $428-433$.

55. Doan, N., P. Reher, S. Meghii and M. Harris, In vitro effects of therapeutic ultrasound on cell proliferation, protein synthesis, and cytokine production by human fibroblasts, osteoblasts, and monocytes. J Oral Maxillofac Surg, 1999. 57(4): p. 40919; discussion 420. 
56. Vanable, J., Integumentary potentials and wound healing, in Electric Fields in Vertebrate Repair, R. Borgens, Editor. 1989, Alan Liss Inc: New York. p. 171-224.

57. Byl, N.N., L. Hill Toulouse, P. Sitton, J. Hall and R. Stern, Effects of ultrasound on the orientation of fibroblasts: an in-vitro study. Eur-J-Phys-MedRehabil, 1996. 6(6): p. 180-4.

58. Demir, H., P. Menku, M. Kirnap, M. Calis and I. Ikizceli, Comparison of the effects of laser, ultrasound, and combined laser plus ultrasound treatments in experimental tendon healing. Lasers Surg. Med., 2004. 35(1): p. 84-89.

59. Tsai, W.C., J.H. Pang, C.C. Hsu, N.K. Chu, M.S. Lin and C.F. Hu, Ultrasound stimulation of types I and III collagen expression of tendon cell and upregulation of transforming growth factor beta. J Orthop Res, 2006. 24(6): p. 1310-6.

60. Yeung, C.K., X. Guo and Y.F. Ng, Pulsed ultrasound treatment accelerates the repair of Achilles tendon rupture in rats. J Orthop Res, 2006. 24(2): p. 193-201.

61. Robertson, V.J., Dosage and treatment response in randomised clinical trials of therapeutic ultrasound. Physical Therapy in Sport, 2002. 3: p. 124-133.

62. Warden, S., K. Bennell, J.M. McMeeken and J. Wark, Can conventional therapeutic ultrasound units be used to accelerate fracture repair? Phys Ther Rev, 1999. 4: p. 117-126.

63. Warden, S.J., R.K. Fuchs, C.K. Kessler, K.G. Avin, R.E. Cardinal and R.L. Stewart, Ultrasound produced by a conventional therapeutic ultrasound unit accelerates fracture repair. Phys Ther, 2006. 86(8): p. 1118-27.

64. McCabe, M. and S. Pye, Therapeutic Ultrasound: Risk associated with poor calibration. Physiotherapy, 1997. 83(5): p. 228.

65. Sutton, Y., K. McBride and S. Pye, An ultrasound mini-balance for measurement of therapy level ultrasound. Phys Med Biol, 2006. 51(14): p. 3397-404.

66. Schabrun, S., L. Chipchase and H. Rickard, Are therapeutic ultrasound units a potential vector for nosocomial infection? Physiother Res Int, 2006. 11(2): p. 61-71.

67. Watson, T., Ultrasound Dose Calculations. In Touch, 2002. 101: p. 14-17. 